\title{
MECHANICAL PARAMETERS OF THE SQUEEZE FILM CURVILINEAR BEARING LUBRICATED WITH A PRANDTL FLUID
}

\author{
A. WALICKA* and E. WALICKI \\ University of Zielona Góra, Faculty of Mechanical Engineering \\ ul. Szafrana 4, 65-516 Zielona Góra, POLAND \\ E-mail: A.Walicka@ijame.uz.zgora.pl
}

\begin{abstract}
Based upon a Prandtl fluid flow model, a curvilinear squeeze film bearing is considered. The equations of motion are given in a specific coordinate system. After general considerations on the Prandtl fluid flow these equations are used to derive the Reynolds equation. The solution of this equation is obtained by a method of successive approximation. As a result one obtains formulae expressing the pressure distribution and load-carrying capacity. The numerical examples of the Prandtl fluid flow in gaps of two simple bearings are presented.
\end{abstract}

Key words: modified Reynolds equation, Prandtl fluid, squeeze film, mechanical parameters of bearing, additives.

\section{Introduction}

Steady laminar flows of a Newtonian fluid in a gap of the bearing with impermeable surfaces have been examined theoretically and experimentally. The bearing walls have been modelled as two cylindrical surfaces (for journal bearings), two disks, two conical or spherical surfaces. A more general case is established by the bearing formed by two surfaces of revolution [1-3].

With the development of modern machine elements the use of complex fluids as lubricants has become of great interest. They also reduce the coefficient of friction and increase the temperature range in which the bearing can operate.

The development of physico-chemistry has led to the introduction of new lubricants which enhance the conditions of the bearing operation and its performance. The mono and multigrade lubricants used in present-day tribological applications are blended with additives which are polymeric in nature. This blending results in a deviation of lubricant rheology from Newtonian to non-Newtonian.

For a long time the non-Newtonian fluids have evoked a considerable interest (see e.g. [4-7]). The complex rheology of biological fluids has motivated investigations involving different non-Newtonian fluids. In recent years, non-Newtonian fluids have become more and more important industrially. Polymer solutions, polymer melts, paints and slurries, shampoo, toothpaste, clay coating and suspensions, greases and cosmetics are the most common examples of non-Newtonian fluids. The property of these fluids is that the stress tensor is related to the deformation tensor by some non-linear relationship. One of the industrial applications of the non-Newtonian fluids is exactly tribology in which such new lubricants as alloys and polymer solutions, electrolytes and liquid metals, ionized gases and ferromagnetic fluids are used.

Studies done on fluid film lubrication have employed several models such as the micropolar [8], couple-stress [2], viscoplastic [9, 10], pseudo-plastic model [11-14] and mixture [15]. Naturally, this list is not complete and given only to present the possibility of mathematical modelling. A more complete list can be found in [1-3].

\footnotetext{
* To whom correspondence should be addressed
} 
This paper is mainly concerned with the non-Newtonian effects in lubrication of a thrust curvilinear bearing lubricated with a Prandtl fluid whose model is given as follows [16]

$$
\tau=\mu\left[\frac{\arcsin (k \dot{\gamma})}{(k \dot{\gamma})}\right] \dot{\gamma}
$$

where $\tau$ is the shear stress, $\mu$ is the coefficient of viscosity, $k$ is the material constant, $\dot{\gamma}$ is the shear strain rate.

In what follows we will take into account only such values of $k$ for which the expression $(k \dot{\gamma})$ satisfies the relationship (Walicka [1,2])

$$
(k \dot{\gamma})<1
$$

we can present the model Eq.(1.1) in the form of a series

$$
\tau=\mu\left[1+\frac{(k \dot{\gamma})^{2}}{2 \cdot 3}+\frac{1 \cdot 3(k \dot{\gamma})^{4}}{2 \cdot 4 \cdot 5}+\ldots\right] \dot{\gamma}
$$

For a sufficiently small value of $(k \dot{\gamma})$ it suffices to limit the expression in brackets of Eq.(1.3) to the first two terms

$$
\tau=\mu\left[1+\frac{(k \dot{\gamma})^{2}}{6}\right] \dot{\gamma}
$$

This formula will be used to model the Prandtl fluid flow in a squeeze film bearing.

\section{Equations of motion of the Prandtl fluid}

The three-dimensional form of the constitutive Eq.(1.4) of the Prandtl fluid is as follows (Walicka [1, 2])

$$
\boldsymbol{T}=-p \boldsymbol{1}+M \boldsymbol{A}_{1}, \quad M=\mu\left(1+\frac{k^{2}}{6} A^{2}\right) \quad A=\left[\frac{1}{2} \operatorname{tr}\left(\boldsymbol{A}_{1}^{2}\right)\right]^{1 / 2}
$$

where 1 is the unit tensor, $p$ is the pressure, $A$ is the second invariant of the stretching tensor $\boldsymbol{A}_{1}$ (the first Rivlin-Ericksen tensor) defined by

$$
\boldsymbol{A}_{1}=\boldsymbol{L}+\boldsymbol{L}^{T}, \quad \boldsymbol{L}=\operatorname{grad} \mathbf{v}
$$

and $\mathbf{v}$ is the velocity vector and $T$ denotes the transposition.

The general equations of motion of the Prandtl fluid have the following forms:

- equation of continuity

$$
\operatorname{div} \mathbf{v}=0
$$


- equation of momentum

$$
\rho \frac{d \mathbf{v}}{d t}=\operatorname{div} \boldsymbol{T}
$$

or

$$
\rho \frac{d \mathbf{v}}{d t}=-\nabla p+\operatorname{div} \boldsymbol{\Lambda}
$$

where

$$
\Lambda=M A_{l} .
$$

Let us consider a thrust bearing with a curvilinear profile of the working surfaces shown in Fig.1. The lower fixed surface is described by the function $R(x)$ which denotes the radius of this surface. The bearing clearance thickness is given by the function $h(x, t)$.

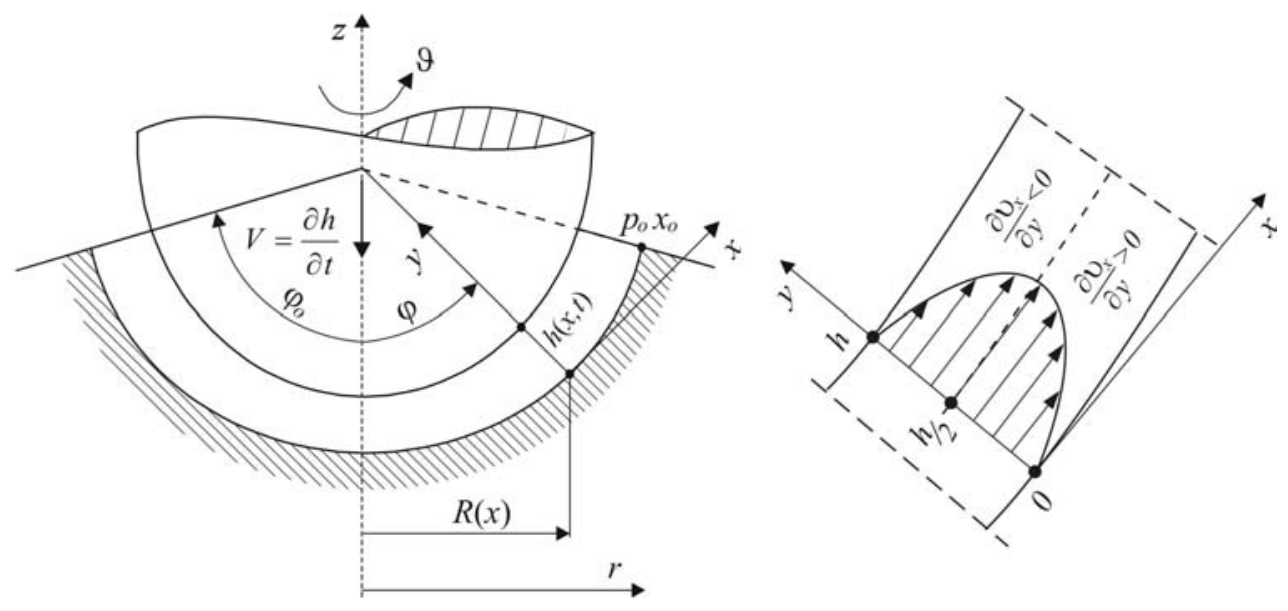

Fig.1. Geometry of a curvilinear bearing.

An intrinsic curvilinear orthogonal coordinate system $x, \vartheta, y$ linked with the lower bearing surface is also presented in Fig.1. The physical parameters of the Prandtl fluid flow are the velocity components $v_{x}, v_{y}$, pressure $p$. With regard to the axial symmetry of the flow these parameters do not depend on the angle $\vartheta$.

The assumption typical for the flow in a narrow gap (Walicka [2], Walicki [3])

$$
h(x)<<R(x)
$$

can be used to make order-of-magnitude arguments for Eqs (2.3)-(2.6).

A further simplification comes by noting that - in accordance with the lubrication approximation the most important changes in an annular bearing channel occur in the normal direction to the main flow. This leads to the assumption that the flow is nearly parallel to the surfaces bounding the gap, so that

$$
v_{y}<<v_{x}, \quad \frac{\partial}{\partial x}<<\frac{\partial}{\partial y}
$$

in an intrinsic coordinate system. 
If some asymptotic transformations are made, the same as in (Walicka [2], Walicki [3]) these equations can be reduced to a simpler form

$$
\begin{aligned}
& \frac{1}{R} \frac{\partial\left(R v_{x}\right)}{\partial x}+\frac{\partial v_{y}}{\partial y}=0 \\
& \rho\left(v_{x} \frac{\partial v_{x}}{\partial x}+v_{y} \frac{\partial v_{x}}{\partial y}\right)=-\frac{\partial p}{\partial x}+\frac{\partial}{\partial y}\left\{\left[\mu+\frac{\mu k^{2}}{6}\left|\frac{\partial v_{x}}{\partial y}\right|^{2}\right] \frac{\partial v_{x}}{\partial y}\right\}, \\
& 0=-\frac{\partial p}{\partial y}
\end{aligned}
$$

The last equation yields

$$
p=p(x, t)
$$

For the slow flows the inertia effects are very small and Eq.(2.8) may be rewritten in the form

$$
\frac{1}{\mu} \frac{d p}{d x}=\frac{\partial^{2} v_{x}}{\partial y^{2}}+\frac{k^{2}}{2}\left|\frac{\partial v_{x}}{\partial y}\right|^{2} \frac{\partial^{2} v_{x}}{\partial y^{2}}
$$

The problem statement is complete after specification of boundary conditions. These conditions for velocity component are stated as follows:

$$
\begin{aligned}
& v_{x}(x, 0, t)=0, \quad v_{x}(x, h, t)=0, \\
& v_{y}(x, 0, t)=0, \quad v_{y}(x, h, t)=\frac{\partial h}{\partial t}=\dot{h} ;
\end{aligned}
$$

the boundary conditions for the pressure distribution will be presented in the next section.

\section{Solution to the equations of motion}

Let us consider the assumption (1.2) which may be presented in the following form

$$
\frac{k^{2}}{2}\left|\frac{\partial v_{x}}{\partial y}\right|^{2}<1
$$

it results that

$$
\beta=\frac{k^{2}}{2}<<1
$$

These relationships make it possible to search for solution of Eq.(2.11) in a series form

$$
v_{x}=\beta^{0} v_{0}+\beta^{1} v_{1}+\beta^{2} v_{2}+\ldots
$$


Putting (3.3) into (2.11) we find for the two first terms

$$
\frac{1}{\mu} \frac{\partial p}{\partial x}=\frac{\partial^{2} v_{0}}{\partial y^{2}}+\beta\left[\frac{\partial^{2} v_{1}}{\partial y^{2}}+\left|\frac{\partial v_{0}}{\partial y}\right|^{2} \frac{\partial^{2} v_{0}}{\partial y^{2}}\right]
$$

Solving this equation for each of the $\beta$ powers $\left(\beta^{0}\right.$ and $\left.\beta^{l}\right)$ and taking into account the boundary conditions (2.12) we will obtain

$$
\mathrm{v}_{x}=\left(-\frac{1}{2 \mu} \frac{\partial p}{\partial x}\right)\left(h y-y^{2}\right)+\frac{k^{2}}{48}\left[|h-2 y|^{4}-h^{4}\right]\left(-\frac{1}{2 \mu} \frac{\partial p}{\partial x}\right)^{3} .
$$

To find the Reynolds equation it is necessary to introduce Eq.(3.5) into Eq.(2.7) and integrate them across the gap thickness. After integration it we have

$$
\frac{1}{R} \frac{\partial}{\partial x} R h^{3}\left[\frac{\partial p}{\partial x}+\frac{k^{2} h^{2}}{40 \mu^{2}}\left(-\frac{\partial p}{\partial x}\right)^{3}\right]=12 \mu \frac{\partial h}{\partial t}
$$

To find the solution to the Reynolds equation (3.6) let us return to the assumption (1.2); it can be use to search the solution of Eq.(3.6) in the form of a sum

$$
p=p^{(0)}+p^{(1)} \text {. }
$$

Assuming that $p^{(1)} \ll p^{(0)}$ and substituting Eq.(3.7) into Eq.(3.6) we arrive at two linearized equations:

- the first one

$$
\frac{1}{R} \frac{\partial}{\partial x} R h^{3} \frac{\partial p}{\partial x}=12 \mu \frac{\partial h}{\partial t}
$$

- and the other

$$
\frac{1}{R} \frac{\partial}{\partial x} R h^{3} \frac{\partial p^{(1)}}{\partial x}=\frac{1}{R} \frac{\partial}{\partial x} \frac{k^{2} R h^{5}}{40 \mu^{2}}\left(\frac{\partial p^{(0)}}{\partial x}\right)^{3}
$$

The boundary conditions for the pressure in a squeeze film are

$$
\left.\frac{\partial p^{(0)}}{\partial x}\right|_{x=0}=0, \quad p^{(0)}\left(x_{o}\right)=p_{o},\left.\quad \frac{\partial p^{(l)}}{\partial x}\right|_{x=0}=0, \quad p^{(l)}\left(x_{o}\right)=0
$$

The solutions of Eqs (3.8) and (3.9) are given as follows

$$
p(x, t)=p_{o}-12 \mu\left[F_{o}-F(x, t)\right]
$$

where 


$$
\begin{aligned}
& F(x, t)=I(x, t)+\frac{18}{5} k^{2} J(x, t), \quad F_{o}=F\left(x_{o}, t\right), \\
& I(x, t)=\int \frac{\int R \dot{h} d x}{R h^{3}} d x, \quad J(x, t)=\int \frac{\left(\int R \dot{h} d x\right)^{3}}{R^{3} h^{7}} d x,
\end{aligned}
$$

The load-carrying capacity is defined by

$$
N=2 \pi \int_{0}^{x_{o}}\left(p-p_{o}\right) R \cos \varphi d x
$$

the sense of the angle $\varphi$ arises from Fig.1.

\section{Thrust squeeze film bearings}

Let us consider a radial thrust bearing with a squeezed film of lubricant modelled by two parallel disks (Fig.8).

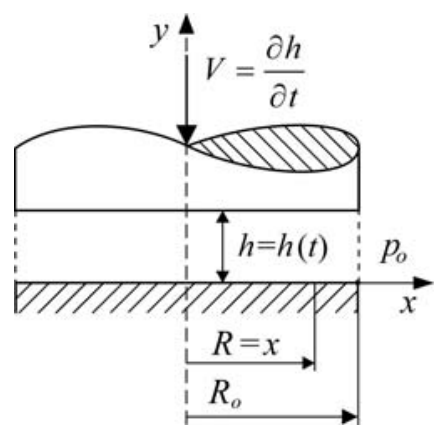

Fig.2. Squeeze film in a radial thrust bearing.

Introducing the following parameters

$$
\begin{aligned}
& R=x, \quad \tilde{x}=\frac{x}{x_{o}}, \quad \tilde{h}=\frac{h}{h_{o}}=e(t), \quad e(t)=1-\varepsilon(t), \quad \dot{\varepsilon}=\frac{d \varepsilon}{d t}, \\
& \tilde{p}=\frac{\left(p-p_{o}\right)}{\mu \dot{\varepsilon}}\left(\frac{h_{o}}{x_{o}}\right)^{2}, \quad \tilde{N}=\frac{N h_{o}^{2}}{\mu \dot{\varepsilon} x_{o}^{4}}, \quad \lambda_{p}=k^{2}\left(\frac{\dot{\varepsilon} x_{o}}{h_{o}}\right)^{2}
\end{aligned}
$$

we will obtain the formulae for the dimensionless pressure distribution and load-carrying capacity for the radial thrust bearing with a squeeze film of the lubricant

$$
\tilde{p}=\frac{3}{e^{3}}\left[1-\tilde{x}^{2}+\frac{9}{20} \frac{\lambda_{p}}{e^{4}}\left(1-\tilde{x}^{4}\right)\right],
$$




$$
\tilde{N}=\frac{3 \pi}{2 e^{3}}\left(1+\frac{3 \lambda_{p}}{5 e^{4}}\right)
$$

Plots of the dimensionless pressure distribution and load-carrying capacity are presented in Figs 3 and 4.

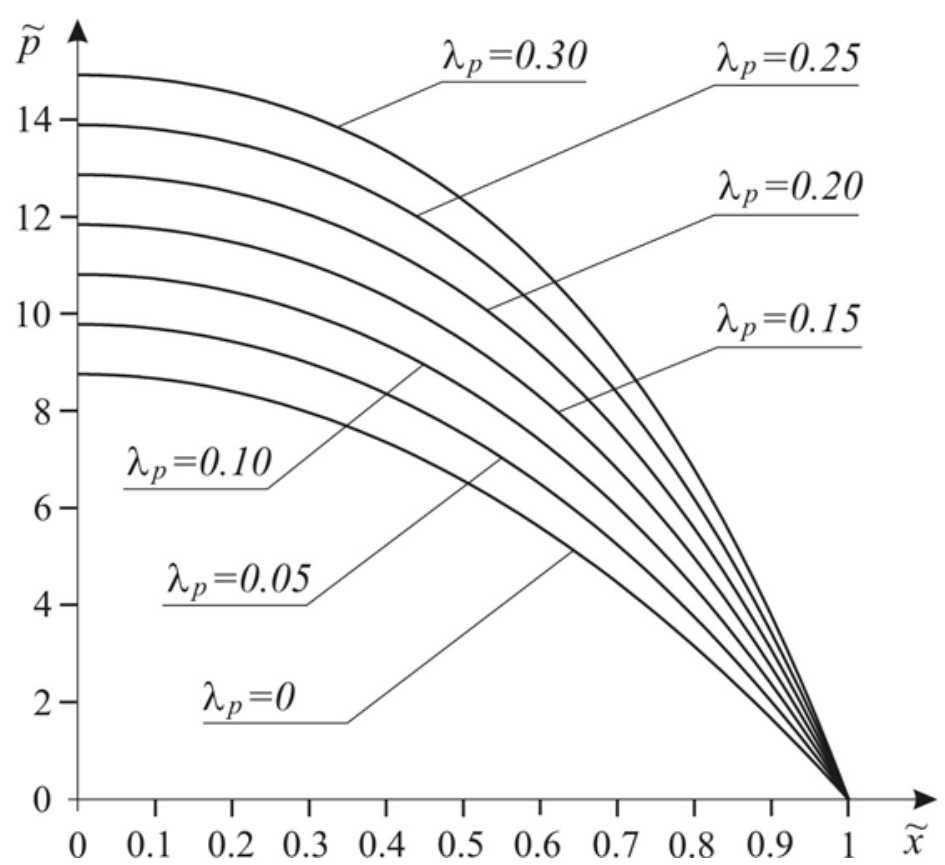

Fig.3. Dimensionless pressure distribution in the radial squeeze film bearing for $\varepsilon=0.3$ versus different values of $\lambda_{p}$.

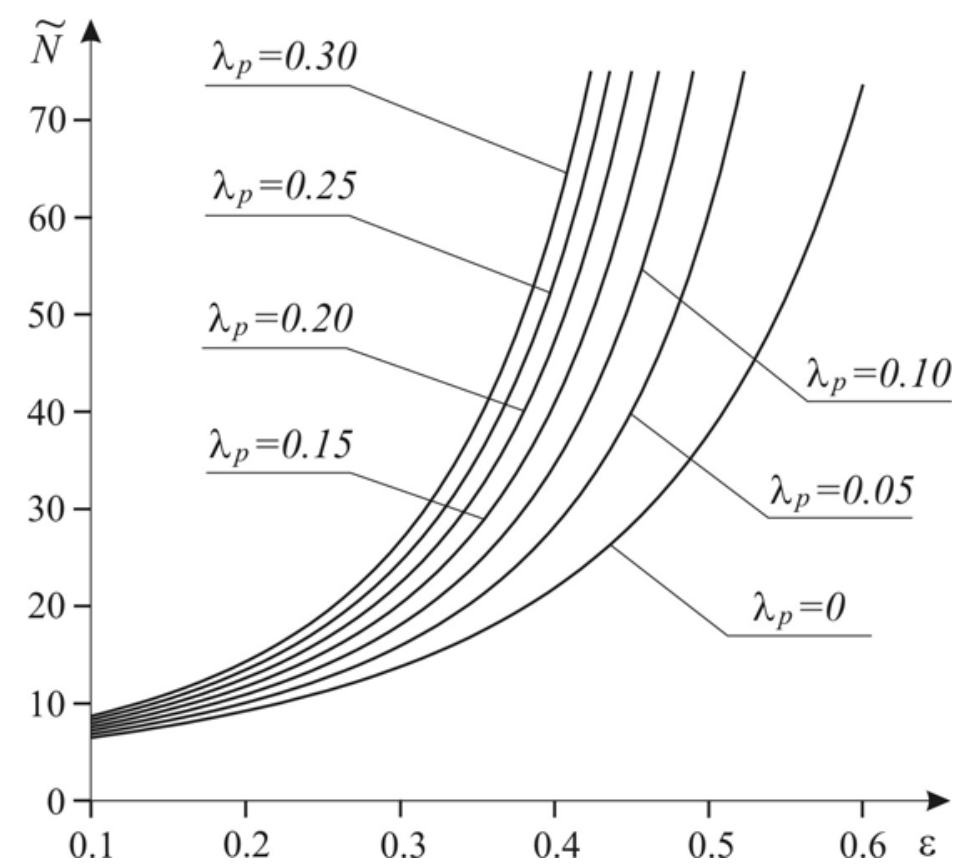

Fig.4. Dimensionless load-carrying capacity in the radial squeeze film bearing for different values of $\lambda_{p}$. 
Let us consider now a spherical squeeze film bearing shown in Fig.5.

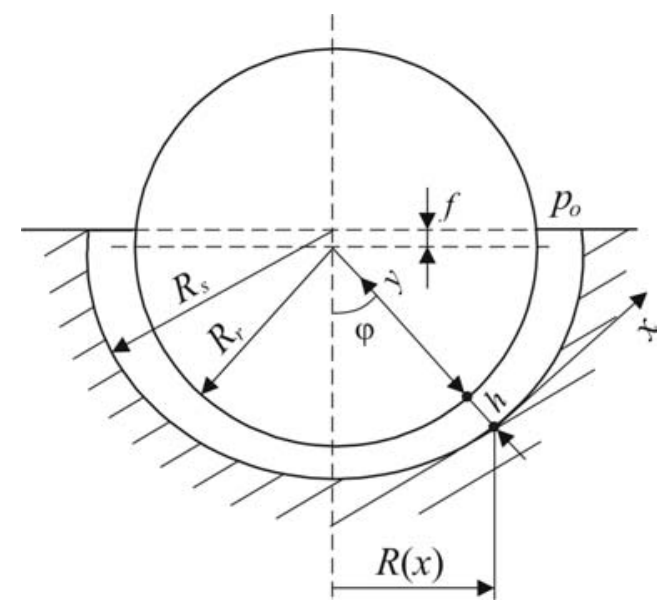

$$
\begin{aligned}
& C=R_{s}-R_{r}, \\
& \varepsilon(t)=\frac{f}{C}, \\
& \varphi=\frac{x}{R_{r}}, \\
& R=R_{S} \sin \varphi .
\end{aligned}
$$

Fig.5. Spherical squeeze film bearing $\left(\right.$ for $\left.\varphi_{o}=\frac{\pi}{2}\right)$.

Introducing the following parameters

$$
\begin{aligned}
& u=\tilde{h}=\frac{h}{C}=1-\varepsilon \cos \varphi, \quad u_{o}=1-\varepsilon \cos \varphi_{o}, \quad e=1-\varepsilon, \quad \Phi=u_{o}^{2}-e^{2}-2\left(u_{o}-e\right), \\
& \tilde{p}=\frac{\left(p-p_{o}\right)}{\mu \dot{\varepsilon}}\left(\frac{C}{R_{r}}\right)^{2}, \quad \tilde{N}=\frac{N C^{2}}{\mu \dot{\varepsilon} R_{r}^{4}}, \quad \lambda_{p}=k^{2}\left(\frac{\dot{\varepsilon} R_{r}}{C}\right)^{2},
\end{aligned}
$$

we will obtain the formulae for the dimensionless pressure distribution and load-carrying capacity for the spherical squeeze film bearing

$$
\begin{aligned}
& \tilde{p}=-\frac{3}{\varepsilon}\left\{\left(\frac{1}{u_{o}^{2}}-\frac{1}{u^{2}}\right)-\frac{15}{32} \frac{\lambda_{p}}{\varepsilon^{2}}\left[\left(1-\varepsilon^{2}\right)\left(\frac{1}{u_{o}^{6}}-\frac{1}{u^{6}}\right)+\right.\right. \\
& \left.\left.-\frac{12}{5}\left(\frac{1}{u_{o}^{5}}-\frac{1}{u^{5}}\right)+\frac{3}{2}\left(\frac{1}{u_{o}^{4}}-\frac{1}{u^{4}}\right)\right]\right\}, \\
& \tilde{N}=-\frac{6 \pi}{\varepsilon^{3}}\left(\ln u_{o}-\ln e+\frac{1}{u_{o}}-\frac{1}{e}-\frac{\Phi}{2 u_{o}^{2}}-\frac{15}{32} \frac{\lambda_{p}}{\varepsilon^{2}}\left\{( 1 - \varepsilon ^ { 2 } ) \left[\frac{1}{5}\left(\frac{1}{u_{o}^{5}}-\frac{1}{e^{5}}\right)+\right.\right.\right. \\
& \left.-\frac{1}{4}\left(\frac{1}{u_{o}^{4}}-\frac{1}{e^{4}}\right)-\frac{\Phi}{2 u_{o}^{6}}\right]-\frac{12}{5}\left[\frac{1}{4}\left(\frac{1}{u_{o}^{4}}-\frac{1}{e^{4}}\right)-\frac{1}{3}\left(\frac{1}{u_{o}^{3}}-\frac{1}{e^{3}}\right)-\frac{\Phi}{2 u_{o}^{5}}\right]+ \\
& \left.\left.+\frac{3}{2}\left[\frac{1}{3}\left(\frac{1}{u_{o}^{3}}-\frac{1}{e^{3}}\right)-\frac{1}{2}\left(\frac{1}{u_{o}^{2}}-\frac{1}{e^{2}}\right)-\frac{\Phi}{2 u_{o}^{4}}\right]\right\}\right) .
\end{aligned}
$$


Plots of the dimensionless pressure distribution and load-carrying capacity are presented in Figs 6 and 7 .

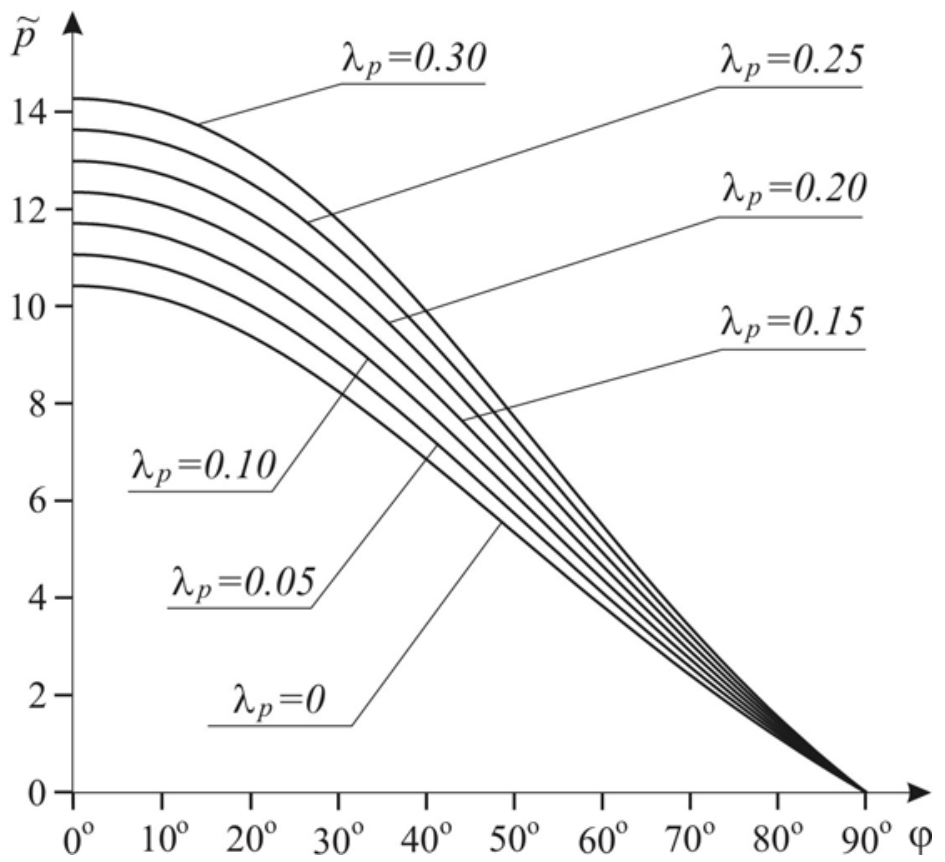

Fig.6. Dimensionless pressure distribution in the spherical squeeze film bearing for $\varepsilon=0.3$ versus different values of $\lambda_{p}$.

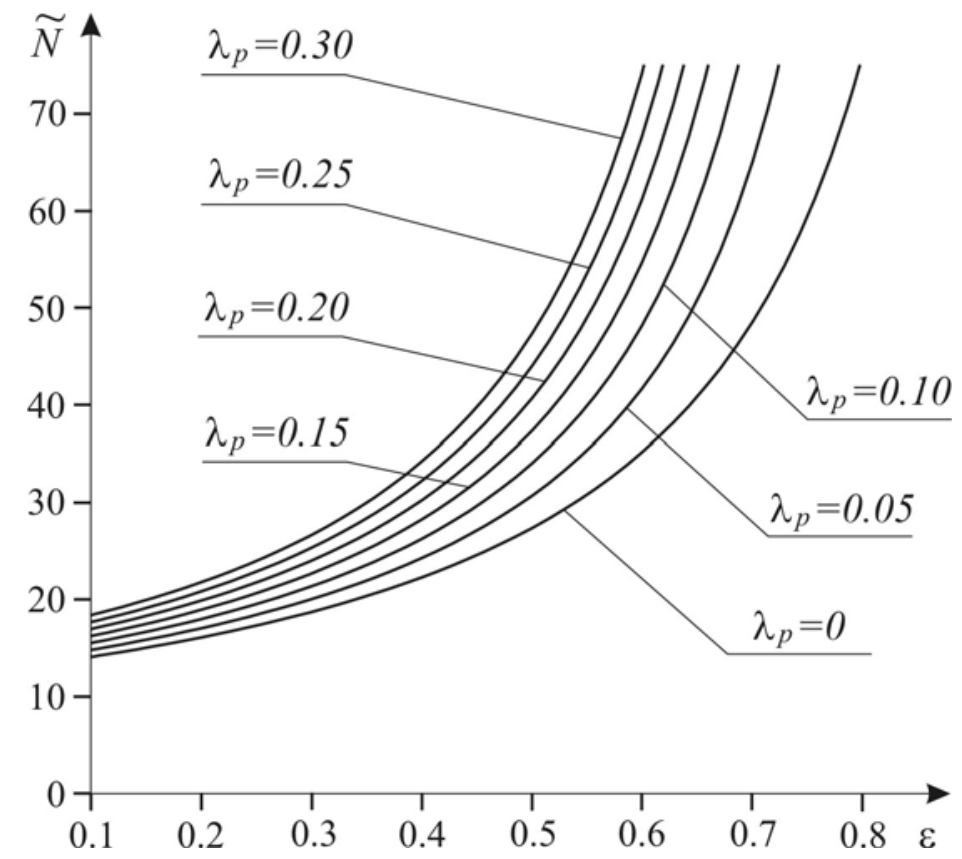

Fig.7. Dimensionless load-carrying capacity in the spherical squeeze film bearing for different values of $\lambda_{p}$.

Note that the case of $\lambda_{p}=0$ represents the pressure distributions and load-carrying capacities for the flow of a Newtonian lubricant. 
It is easy to see from the presented plots that the increase of the values of $\lambda_{p}$ evokes a significant increase in the values of the mechanical parameters of both the bearings considered here. This increase is more visible in the gap of the radial bearing with respect to that one in the gap of the spherical bearing.

\section{Conclusion}

The modified Reynolds equation for a Prandtl type of a pseudo-plastic lubricant flowing in a gap of a thrust curvilinear bearing with squeeze film is derived. As a result the general formulae for pressure distribution and load-carrying capacity are obtained. It follows from the calculations carried out and their graphic presentations that both the magnitudes depend on the values of rheological parameters $k$ or $\lambda_{p}$.

It may be concluded that in both the bearings under consideration the pressures and load-carrying capacities increase with the increase of $\lambda_{p}$. These increases are more visible for the radial squeeze film bearing.

\section{Nomenclature}

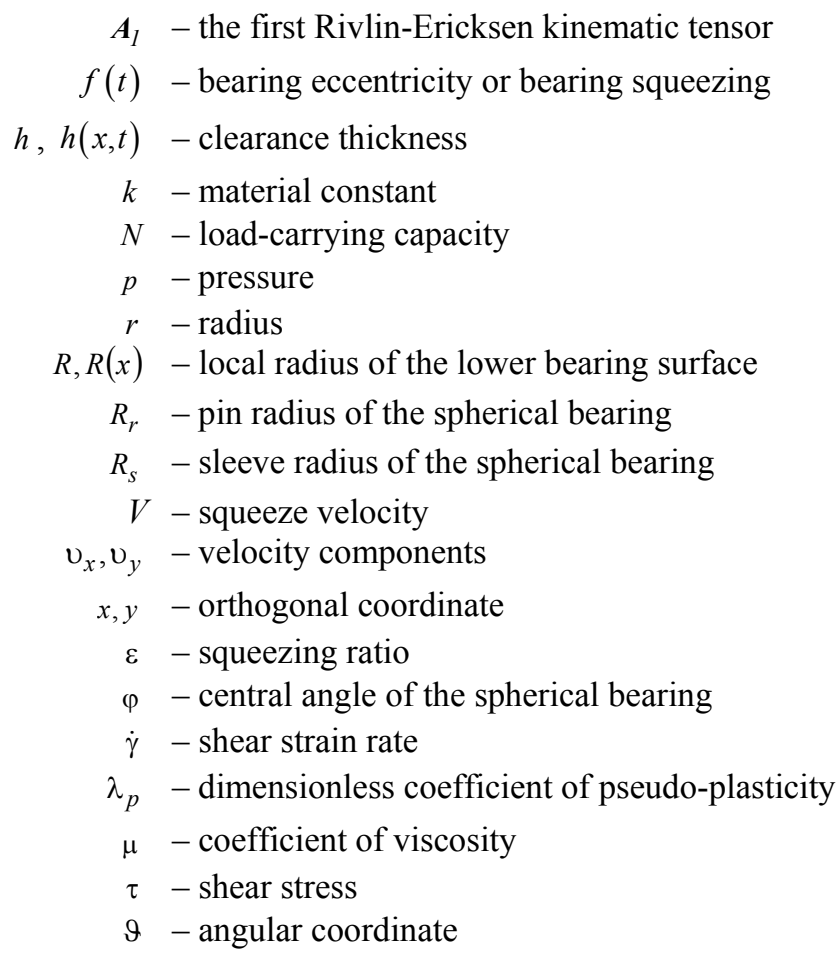

\section{References}

[1] Walicka A. (2002a): Rheodynamics of Non-Newtonian Fluids Flow in Straight and Curved Channels (in Polish). Zielona Gora: University Press.

[2] Walicka A. (2002b): Rotational Flows of Rheological Complex Media in Narrow Annular Channels (in Russian). Zielona Góra: University Press.

[3] Walicki E. (2005): Rheodynamics of Slide Bearings Lubrication (in Polish). - Zielona Gora: University Press.

[4] Bird R.B., Stewart W.E. and Lightfoot F.W. (1960): Transport Phenomena. - New York: John Wiley.

[5] Wilkinson W.L. (1960): Non-Newtonian Fluid. - New York: Pergamon. 
[6] Metzner A.B. (1965): Heat transfer in non-Newtonian fluids. - Adv. Heat Transfer, vol.2, pp.357-397.

[7] Skelland A.H.P. (1967): Non-Newtonian Flow and Heat Transfer. - New York: John Wiley.

[8] Walicka A. (1994): Micropolar Flow in a Slot Between Rotating Surfaces of Revolution. - Zielona Góra: TU Press.

[9] Lipscomb C.C. and Denn M.M. (1984): Flow of Bingham fluids in complex geometries. - J. Non-Newt. Fluid Mech., vol.14, No.3, pp.337-349.

[10] Dorier C. and Tichy J. (1992): Behaviour of a Bingham-like viscous fluid in lubrication flows. - J. Non-Newt. Fluid Mech., vol.45, No.3, pp.291-350.

[11] Wada S. and Hayashi H. (1971a): Hydrodynamic lubrication of journal bearings by pseudo-plastic lubricants, (Pt 1, Theoretical studies). - Bull. JSME, vol.14, No.69, pp.268-278.

[12] Wada S. and Hayashi H. (1971b): Hydrodynamic lubrication of journal bearings by pseudo-plastic lubricants. (Pt 2, Experimental studies). - Bull. JSME, vol.14, No.69, pp.279-286.

[13] Swamy S.T.N., Prabhu B.S. and Rao B.V.A. (1975): Stiffness and damping characteristics of finite width journal bearing with a non-Newtonian film and their application to instability prediction. - Wear, vol.32, pp.379-390.

[14] Rajalingham C., Rao B.V.A. and Prabu S. (1978): The effect of a non-Newtonian lubricant on piston ring lubrication. - Wear, vol.50, pp.47-57.

[15] Khonsari M.M. and Dai F. (1992): On the mixture flow problem in lubrication of hydrodynamic bearing: small solid volume fraction. - STLE Trib. Trans., vol.35, No.1, pp.45-52.

[16] Prandtl L. (1928): Ein Gedankenmodell zur kinetischen Theorie der festen Körper. - ZAMM, vol.8, No.2, pp.85107.

Received: May 23, 2016

Revised: June 12, 2016 